\title{
Carnets
}

Revue électronique d'études françaises de l'APEF

Première Série - 4 Numéro Spécial | 2012

Invasions \& Évasions

\section{La France, terre d'exil et d'évasion dans Square Tolstoi de Nuno Bragança}

Isabelle Simões Marques

\section{OpenEdition}

1 Journals

Edição electrónica

URL: http://journals.openedition.org/carnets/7866

DOI: $10.4000 /$ carnets.7866

ISSN: 1646-7698

\section{Editora}

APEF

Edição impressa

Data de publição: 1 Junho 2012

Paginação: 275- 289

Refêrencia eletrónica

Isabelle Simões Marques, "La France, terre d'exil et d'évasion dans Square Tolstoi de Nuno Bragança », Carnets [Online], Première Série - 4 Numéro Spécial | 2012, posto online no dia 23 junho 2018,

consultado o 01 maio 2019. URL : http://journals.openedition.org/carnets/7866 ; DOI : 10.4000/ carnets.7866

\section{(c) (1) (8)}

Carnets est mis à disposition selon les termes de la licence Creative Commons - Atribution - Pas d'utilisation commerciale 4.0 International. 


\title{
LA FRANCE TERRE D'EXIL ET D'ÉVASION DANS SQUARE TOLSTOI DE NUNO BRAGANÇA
}

\author{
ISABELLE SIMÕES MARQUES \\ Universidade de Coimbra \\ Centro de Linguística da Universidade Nova de Lisboa \\ Equipe d'accueil Laboratoire d' Études romanes, Université Paris 8 \\ isabelle@fl.uc.pt
}

\begin{abstract}
Resumo
O escritor migrante, que possui na sua escrita um terreno fértil para a sua produção literária, exorciza de alguma forma a sua existência. A escrita de Bragança inclui a sua condição de dualidade : por um lado, a função referencial expressa em factos reais que são apresentados e, por outro lado, a função poética expressa em sua obra. Veremos que existem no romance marcas de alteridade na linguagem destinada a revelar o plurilinguismo do autor. Bragança usa termos, citações e referências da língua francesa. O discurso do Outro tem por base o princípio da alteridade radical, da heterogeneidade e da exterioridade irredutível que inclui também a do autor, que destaca a língua e a cultura francesas no seu romance. O nosso objectivo é a análise das funções discursiva, estilística e expressiva da presença francesa em todo o romance e, assim, identificar as suas principais implicações na dinâmica do texto.
\end{abstract}

\section{Abstract}

The migrant writer who has a fertile ground for literary production in his writing, somehow manages to exorcise his own existence. The writing of Bragança includes his condition of duality : on the one hand, we are presented with the referential function expressed in actual facts, and on the other, we have the poetic function expressed in his novel. The novel presents us with marks of otherness in the language designed to reveal the author's multilingualism. Bragança uses terms, quotations and references to the French language. The discourse of the Other is based on the principle of radical otherness, heterogeneity and irreducible exteriority which also includes the author, giving a great emphasis to the French language and culture in his novel. Our goal is the analysis of discourse, stylistic and expressive functions regarding the French presence in the whole novel, and thus identifying their major implications on the text's dynamics.

Palavras-chave: Plurilinguismo, exílio, alteridade, romance autobiográfico.

Keywords: Multilingualism, exile, otherness, autobiographical novel. 
Introduction

Nuno Manuel Maria Caupers Bragança est né en 1929 dans une grande famille aristocratique portugaise. II a notamment suivi des études d'agronomie et a obtenu une Maîtrise de Droit en 1958. C'est alors qu'il publie ses premiers textes dans le journal Encontro, journal catholique de l'Université de Lisbonne. Dans les années 1960, ses positions politiques se sont radicalisées. II a été militant du Mouvement d'Action Révolutionnaire et de la Résistance Chrétienne et a développé une action clandestine fondamentale, aidant de nombreuses fuites, de nombreuses actions clandestines ou servant d'intermédiaire. En 1968 il part pour Paris où il est représentant permanent de l'OCDE pour le Portugal jusqu'en 1972 (date où il rentre au Portugal). Pendant cette période, il a pu concilier sa mission à l'OCDE avec ses activités littéraires (son premier roman $A$ noite e 0 riso date de 1969) et son action politique l'a rapproché des Brigades Révolutionnaires. En 1973, il a co-signé une expérience cinématographique, centrée sur le problème de l'émigration portugaise et intitulée Nationalité Portugais. Square Tolstoi (Bragança, 1981), roman publié au Portugal en 1981 raconte, de façon autobiographique, cet épisode de vie parisien de l'auteur. Bragança est décédé en 1985.

Dans Square Tolstoï nous sommes face à une réflexion sur l'écriture et la narration de faits historiques liés à la dictature, dans une intrigue qui se rapporte aux activités clandestines des portugais en exil à travers Aníbal, narrateur et personnage principal. Maria Alzira Seixo considère que l'œuvre croise les filons politiques, érotiques et autobiographiques et qu'elle présente l'Histoire à travers la conscience littéraire : um livro para escrever, uma mulher para amar, um país para libertar" (Seixo, 1983 : 97). Le roman souligne la lutte anti-fasciste, la lutte pour la démocratie, qui n'est possible qu'en dehors des frontières du Portugal.

La question du roman autobiographique

Concernant Square Tolstoi nous considérons qu'il illustre bien ce croisement de l'autobiographique et du romanesque. En effet, Nuno Bragança raconte une histoire qui se fonde sur sa propre expérience personnelle. L'auteur se trouve ainsi à l'intersection de son roman et peut choisir d'assumer son identité ou au contraire de maintenir une certaine ambigüité avec le lecteur. Ainsi, Aníbal est, selon nous, l'alter-ego de Nuno Bragança et le porte-parole de l'auteur. II est, en effet, son double narratif, comme le montre l'extrait suivant : 
A semana seguinte deixou-me em paz. Regressei à disciplina do escrever. Acordava às 5,30 da manhã, pequenalmoçava e saltava para a mesa de trabalho. Às 9,30 punha a correr o banho enquanto me barbeava, quebrantado. Dentro da banheira, sacudia o máximo possível da tensão de ter escrito, mas era sempre confuso - ainda que descia à garagem colectiva buscar a Peugeot de desporto (três carretos, guiador de corrida). Pedalava pelos restos esventrados do que fora o Bosque de Bolonha até chegar à vivenda inglesa estilo Hitchcock, onde trocava tempo e energia irrecuperáveis pelo ganho da minha vida e da dos meus filhos em Lisboa. Almoçava no self da Organização do Capital Unido (OCU), e regressava à sede da Delegação portuguesa junto de tal instância. (Bragança, 1981 : 29) (en italique dans l'original).

(fr.) La semaine suivante m'a laissé en paix. Je suis retourné à la discipline de l'écriture. Je me réveillais à $5 \mathrm{~h} 30$ du matin, je petit-déjeunais et je sautais sur ma table de travail. À 9 h30 je faisais couler mon bain pendant que je me rasais, accablé. Dans la baignoire, je secouais un maximum de la tension d'avoir écrit, mais c' était toujours confus, même si je descendais au parking collectif chercher ma Peugeot sportive (trois pignons, un guidon de course). Je pédalais à travers les restes éventrés de ce qui avait été le Bois de Boulogne pour atteindre la villa anglaise au style Hitchcockien, où j'échangeais du temps et de l'énergie irrécupérables par le gain de ma vie et celle de mes enfants à Lisbonne. Je déjeunais au self de I'Organisation du Capital Uni $(\mathrm{OCU})$, et je retournais au siège de la Délégation portugaise auprès de cette instance. (traduction faite par nos soins).

Le narrateur, Aníbal, décrit ici son quotidien parisien, partagé entre son travail d'écriture et ses fonctions à la délégation portugaise de la OCU (OCDE dans la réalité).

Dans l'extrait suivant, le narrateur décrit ses activités de lutte clandestine contre le régime dictatorial, ce qui correspond à ce que nous savons de la biographie de l'auteur.

Às três da tarde do dia seguinte, sexta-feira, reencontrei o Hugo que me passou uma pasta castanha, com fechos doiradinhos. Nessa manhã eu tinha-me arrancado a muito custo dum sono de duas horas para recontactar a "Irene" e o camarada que abordaria a Marta para Ihe passar o material, segundo um esquema combinado com rigor. (Bragança, $1981: 86$ )

(fr.) À trois heures de l'après-midi du jour suivant, vendredi, j'ai rencontré à nouveau Hugo qui m'a passé un portefeuille marron aux fermetures dorées. Ce matin-là, j'étais sorti avec difficulté d'un sommeil de deux heures pour recontacter "Irene" et le camarade qui aborderait Marta pour lui passer le matériel, selon un schéma monté avec soin. 
Aníbal révèle ainsi être l'intermédiaire et le "facteur " de la lutte clandestine à Paris. II décrit avec précision les rendez-vous clandestins et le transfert de documents.

Ce roman constitue donc un récit de vie personnel où sont retracées les trajectoires et les réflexions de l'auteur. Ceci d' autant plus que l'écriture du roman survient avec une certaine distance temporelle des événements vécus. En effet, Square Tolstoi n'a été rédigé qu'à partir de 1979 au Portugal et publié à Lisbonne en 1981. Ce roman est constitué de différents épisodes de la vie de l'auteur et le roman autobiographique permet cette rétrospection assumée par le narrateur.

Or, cette posture associée au moi et à l'écriture est en effet symptomatique de l'époque contemporaine : pour exprimer dans des formes nouvelles leur vécu et faire entendre leur voix, de nombreux écrivains adoptent une attitude "déconstructionniste" vouée à mettre en cause les concepts de vérité, d'authenticité et de fictionnalité (Voir Leblanc 2004 : 10).

\section{La question de l'exil}

Si, comme le précise Claude Lévi-Strauss (1983), c'est à partir des autres que l'on se forge sa propre identité, nous pouvons considérer que le XXe siècle est propice à cette réflexion. Ceci est le résultat d'un monde de plus en plus globalisé qui a forgé un nouveau type d'art : un art fait par des exilés ou des expatriés. Des personnes qui, loin de leur culture natale tentent à la fois de maintenir les liens culturels et de refouler les faits qui les ont contraints à quitter leur pays d'origine, de façon temporaire ou définitive, à l'instar de Nuno Bragança qui a quitté le Portugal pendant la dictature. La différence dans la diaspora ne fonctionne pas autour de processus binaires fermés tels que "je suis d'ici " ou "je suis de làbas" ou de frontières, mais plutôt comme des places de passage, toujours en relation, au long d'un spectre sans début ni fin (cf. Stuart Hall, 2004). Ce concept correspond à Square Tolstoi qui se situe dans un entre-deux constant. Ainsi, nous ne cherchons pas seulement à évaluer l'influence de l'exil dans le processus de création littéraire de l'auteur mais nous tentons également de considérer le rôle que la littérature peut jouer dans l'assimilation de l'exil comme forme de vie pour Nuno Bragança. Une œuvre littéraire apporte toujours en son sein, même si ce n'est pas son principal objectif, une gamme d'éléments historiques. La littérature liée à l'exil a comme principale caractéristique l'historicité, étant donné qu'elle a pour objectif l'expression de sentiments et de faits survenus durant un processus d'expatriation. C'est notamment dans le roman - où l'on raconte une histoire - que s'insère la représentation de cette réalité spécifique. La notion de littérature d'exil pose en outre le problème général de la relation entre expatriation, écriture et langue. Ainsi, le langage n'est 
pas le reflet parfait de la réalité transtextuelle mais plutôt la verbalisation du réel dans le monde fictionnel, ceci impliquant un investissement dans l'expérimentation du langage artistique, dans l'innovation discursive et dans la recherche de nouveaux parcours et perspectives pour cette écriture romanesque qui retrace l'Histoire. Cet investissement dans l'expérimentation d'écriture, dans la problématisation autour de la représentation de l'événement historique conditionne et révèle d'autres traits caractéristiques de la fiction portugaise contemporaine, c'est-à-dire la métafictionnalité et l'intertextualité car les romans portugais contemporains ont tendance à unir la textualité à l'autoréférentialité (cf. Seixo, 1986; Roani, 2004). Le narrateur de Square Tolstoi explique ainsi pourquoi il a fait le choix de partir pour vivre et écrire de façon libre et sans contraintes :

Eu estava em condições de cortar com tudo e todos quantos se atravessassem entre a minha pessoa e a minha escrita. Era para isso que tinha abominado emprego em Paris: com ele ganhava o suficiente para viver e manter os filhos em crescimento estudantil. E entretanto, escrever o que não poderia escrever em Portugal. (Bragança, $1981: 44)$

(fr.) J'étais en condition de couper avec tout et tous ceux qui se mettraient entre moi et mon écriture. C'était pour cela que j'avais accepté un emploi à Paris : grâce à cela je gagnais suffisamment pour vivre et éduquer mes enfants. Et pendant ce temps, j'écrivais ce que je ne pouvais pas écrire au Portugal.

Podia ser outra cidade onde houvesse pintura e gente em movimento. Calhou Paris. O básico é que, fora de Portugal, eu ser estrangeiro é normal e tem estatuto. (Bragança, 1981 : 123)

fr.) Cela aurait pu être une autre ville où il y aurait de la peinture et des gens en mouvement. C'est tombé sur Paris. En fait, en dehors du Portugal, que je sois étranger est normal et me donne un certain statut.

L'écriture représente une prise de liberté et un moyen de s'évader de son quotidien au Portugal, qui était, pour lui, étouffant. Le narrateur voit également dans l'écriture le moyen d'effectuer une catharsis personnelle. II assume le rôle pris par l'écriture dans la démarche cathartique, qui n'est possible qu'à l'étranger :

Irritei-me como me irritara com o Jed e dei-te a mesma explicação que a este: o facto de eu estar purgando a memória (no escrever) tinha um efeito de catarse. (Bragança, $1981: 172)$ 
(fr.) Je me suis irrité avec Jed et je te donne la même explication que je lui ai donné à lui : le fait que je purge la mémoire (dans l'écriture) avait un effet cathartique.

Ainsi, un écrivain exilé comme Nuno Bragança est un être de frontière et son écriture comprend sa condition de dualité : d' un côté la fonction référentielle exprimée dans les faits réels qu'il présente et d' un autre côté la fonction poétique exprimée dans son œuvre. De plus, la lecture de ce roman n'est ni immédiate ni consensuelle et cette littérature est investie d'une fonction de dénonciation et d'alerte. Le sujet-narrateur se retrouve dans sa condition d'étranger, face à lui-même et face aux autres. Ainsi, nous sommes face à un roman qui problématise la crise de reconnaissance de l'être personnel et de l'être portugais. C'est ainsi que cette littérature désigne de façon permanente l'Autre.

Cette littérature "nouvelle" a ainsi opéré, à partir des années 1960, des ruptures au niveau du langage en intégrant en son sein des marques d'altérité au moyen de procédés linguistiques visant à révéler le plurilinguisme. Square Tolstoi nous semble significatif d'une époque de transition dans la société portugaise. L'écrivain qui vit entre différentes cultures développe nécessairement une sensibilité particulière concernant l'Autre et produit une littérature profondément liée au brassage des cultures. II nous semble important de souligner que le choix de la langue d' écriture est une question qui touche tout type d' écrivain, qu' il soit expatrié ou non, car tout écrivain doit s' approprier sa langue d' écriture (cf. Kristeva, 1997). Le phénomène de l'expatriation ne vient qu'exacerber la conscience langagière, propice à l'écriture (cf. Huston, 1999). Ainsi, langue maternelle et langue étrangère sont sur le même pied d'égalité, et l'écrivain doit envisager sa langue d'écriture sous un regard autre à fin de percevoir la profusion de langues littéraires qui sont à sa disposition (cf. Ricœur, 2004). De cette façon, l'écriture permet à la fois de prendre conscience que sa langue maternelle n'est pas unique, mais aussi qu'elle fait partie d'une multitude de langues. Ceci participe de la conscience de l'universalité du langage, mais également de la littérature.

L'écriture permet ainsi de donner un visage à l'Autre et à ce qui est différent. L'idée de la différence - opposée à celle d'unité (unité voulue notamment par la dictature au Portugal) - est comprise dans le plurilinguisme et elle est sans doute l'une des traces énonciatives les plus importantes. L'hétéroglossie, c'est-à-dire la coexistence, dans une société donnée et dans un moment donné, d'une multiplicité de dialectes sociaux et professionnels, de styles et de genres littéraires, de rhétoriques, constitue la réalité et la vie du langage. Pour Mikhaïl Bakhtine, le roman est par excellence le lieu d'une polyphonie qui explore l'hétéroglossie ambiante. Le plurilinguisme littéraire, ou plus précisément l'énonciation en langue étrangère, correspond à un mot, une locution ou un passage entier qui appartiennent à une autre langue et qui sont introduits dans le roman. La langue étrangère est un élément textuel parmi d'autres pour signifier le rapport que nous établissons 
entre identité et altérité dans les domaines de l'aliénation qui concerne notamment l'exil. La langue étrangère peut donc faire partie d'un stratagème, d'une façon de se singulariser ou de se démarquer culturellement ou peut servir à créer différents effets littéraires poétiques, soit de dépaysement, soit de désémantisation. L'hétérogénéité du roman est produite par un métissage de paroles étrangères. Nuno Bragança, qui utilise abondamment des termes issus du français a également recours à des citations et des références en langue française.

En ce qui concerne tout d' abord les emprunts linguistiques nous pouvons affirmer qu'ils permettent d'imprimer des tonalités étrangères au texte. En effet, ils concernent des thématiques précises liées à la France. Les emprunts et les interférences participent ainsi d'un plurilinguisme inhérent à ce roman.

\section{Les emprunts linguistiques}

Nous ne citerons que quelques exemples d'emprunts liés à la France et plus particulièrement à Paris comme le nom des quartiers "Seizième, Cinquième",

Isto já se passava no Seizième. (Bragança, 1981 : 189)

(fr.) Ceci se passait déjà dans le Seizième.

Largámos do $\underline{\text { Cinquième, }}$ estacionei junto a um Pub distante daquela zona proibida a portugueses marcados. (p.36)

(fr.) Nous sommes sortis du Cinquième, j'ai stationné à côté d'un Pub éloigné de cette zone interdite aux portugais listés.

A porta era numa rua do Cinquième. (Bragança, $1981: 140)$

(fr.) La porte était dans une rue du Cinquième.

Les boulevards :

Sentado ainda à mesa do restaurante (o Outro, apressado, pagara a sua parte e levantara-se) vi-o, que se afastava no boulevard. (Bragança, 1981 : 172)

(fr.) Encore assis à la table du restaurant (l'Autre, pressé, avait payé sa part et s'était levé), je l'ai vu, qui s'éloignait dans le boulevard.

la o táxi pelo boulevard Suchet quando o mandei parar. (Bragança, $1981: 209$ )

(fr.) Le taxi allait sur le Boulevard Suchet quand je lui ai demandé de s' arrêter. 
Les différents types d'habitation comme "living-atelier", "appartement bourgeoisconfort " ou "château " :

[...] estava num cabide que era uma das peças decorativas do living-atelier ". (Bragança, 1981 : 62) (fr.) [...] il était sur un portemanteau qui était l'un des éléments décoratifs du living-atelier.

Havia plena luz solar a entrar pelas frestas do living-atelier. (Bragança, 1981 : 85)

(fr.) Il y avait un plein soleil qui pénétrait par les fissures de living-atelier.

Mas logo a própria escada falava em Bourgeois-confort. O tipo de apartamento habitual nos ex-palacetes de St. Germain. (Bragança, 1981 : 140)

(fr.) Mais tout de suite l'escalier même faisait référence au Bourgeois-confort. Le type d'appartement habituel dans les anciennes demeures de St. Germain.

"O meu castelo é este " - e apontei o velho Château onde começara a organização OCU, no pós-guerra. (Bragança, $1981: 133$ )

(fr.) "C'est mon château" - et j'ai pointé en direction de l'ancien Château, où l'organisation de l'OCU avait débuté dans l'après-guerre.

Les vins français comme le "beaujolais", le "bordeaux" ou le "champagne" :

Enquanto trabalhavas no fogão e eu bebia um Beaujolais de aperitivo [...]. (Bragança, $1981: 162)$

(fr.) Pendant que tu travaillais à la cuisinière je buvais un Beaujolais en apéritif [...]. "Este deitou mais Bordeus [sic] nos copos e sorriu ". (Bragança, 1981 : 178)

(fr.) Ce dernier a versé un peu plus de Bordeaux dans les verres et a souri.

Fui direito à cantina da OCU, comprei uma garrafa de champanhe. (p.137)

(fr.) Je suis allé directement à la cantine de l'OCU et j' ai acheté une bouteille de champagne.

Les typiques cafés parisiens :

Chegado ao bistrot, vi o Bigodes levantar-se e vir ao meu encontro. (Bragança, 1981 : 186)

(fr.) Quand je suis arrivé au bistrot, j'ai vu le Moustachu se lever et venir vers moi. 
Une deuxième catégorie linguistique de termes étrangers est présente dans le texte et correspond aux interférences linguistiques - qui sont des termes étrangers utilisés de façon individuelle et sporadique par le narrateur (cf. Calvet, 1993). Les cas suivants sont donc la conséquence d'une influence de la langue française comme le terme "Banholas":

[...] a procissão de parvoíce enlatada nas banholas, já todos irritados contra tudo. (Bragança, $1981: 28$ )

(fr.) [...] la procession du ridicule en boîte dans les bagnoles, tous en colère contre tout.

Ce terme qui provient du terme français "bagnoles" a subi une adaptation graphique et conserve le genre féminin. Ce terme, d'un registre nettement familier, se retrouve à des moments de la narration qui fait clairement référence au quotidien français. Avec le terme "pionnaisada" nous sommes face à un participe passé qui provient du terme français "punaise " :

Ficaste um largo tempo olhando a foto de eu-puto, pionnaisada à cabeceira. (Bragança, 1981 : 185)

(fr.) Tu es restée longtemps à regarder la photo de moi-gamin, punaisée sur la table de chevet.

Concernant les verbes, nous rencontrons un cas intéressant d'influence du français sur le portugais comme l' extrait suivant le montre bien :

Duchei-me, sentei-me à janela da cozinha de luz apagada e copo e cigarros. (Bragança, $1981:$ 43)

(fr.) Je me suis douché, je me suis assis à la fenêtre de la cuisine les lumières éteintes avec un verre et des cigarettes.

Le narrateur utilise ici "duchar" à la forme réfléchie telle qu'elle est communément utilisée en français ( "se doucher "), alors que l' utilisation courante en portugais est "tomar duche".

\section{Citations et références}

Au niveau intertextuel, Square Tolstoi comporte des citations littéraires ou artistiques et des références à des auteurs ou artistes français. Les citations sont disséminées le long 
du roman et appartiennent à la catégorie des "citations canoniques " (cf. Bismuth, 2007) car ce sont des citations qui sont démarquées et référencées dans le texte. Dans Square Tolstoi, nous rencontrons de nombreuses citations. En effet, l'auteur accorde une place de choix aux auteurs français. L'extrait suivant est marqué par l'altérité et la proximité ressentie entre le narrateur et les auteurs cités :

Lancei para lá os lúzios e calhou-me acertar num instantâneo do Breton contando anedotas ao Trotsky num bosque mexicano. Imediatamente uma frase veio businarme na memória : "Dites-vous bien que la littérature est un des plus tristes chemins qui mènent à tout. " Ainda deitei um olhar ao Rilke, mas desta vez ele estava-se nas tintas. (Bragança, $1981: 54$ )

(fr.) J'ai sommeillé et par hasard je suis tombé sur un instantané de Breton racontant des anecdotes à Trotsky dans un bois mexicain. Tout de suite une phrase est venue klaxonner à ma mémoire : "Dites-vous bien que la littérature est un des plus tristes chemins qui mènent à tout. " J'ai encore jeté un regard à Rilke, mais cette fois il s'en fichait. (p.54)

Les auteurs, d'horizons différents (Trotsky, Rilke), font émerger chez le narrateur le manifeste d'André Breton, lié au mouvement du surréalisme, mouvement littéraire et artistique à l'ampleur internationale.

Nous rencontrons également une citation de Max Jacob, artiste et écrivain français, mort dans les camps de concentration durant la lle Guerre Mondiale :

Em tempos, apanhara no ar uma frase do Max Jacob que voara por mim, e era esta : “L' univers est une motte de beurre. On n' a qu' a s' y enfoncer sans hésitation ". (Bragança, $1981: 85$ )

(fr.) Une fois, j'ai pris dans l'air une phrase de Max Jacob qui a volé pour moi, et était la suivante :

"L'univers est une motte de beurre. On n'a qu' à s' y enfoncer sans hésitation".

Le narrateur retranscrit ici en français une citation qu'il se réapproprie. Le narrateur de Square Tolstoi fait également référence à Maurice Blanchot, célèbre critique littéraire français, à travers une citation retirée de son essai Le livre à venir (1959) :

Abri a última do tio Blanchot e cacei duas que meti no meu Diário :

1) 'L'œuvre demande que l'homme qui l'écrit se sacrifie pour l'œuvre, devienne non pas un autre, non pas du vivant qu'il était l'écrivain avec ses devoirs, ses satisfactions et ses intérêts, mais plutôt personne, le lieu vide et animé où retentit l'appel de l'œuvre" (Blanchot, Le Livre à Venir) (Bragança, 1981 : 194) 
(fr.) J'ai ouvert la dernière de l'oncle Blanchot et j'en ai attrapé deux que j'ai mis dans mon Journal :

1) 'L'œuvre demande que l'homme qui l'écrit se sacrifie pour l'œuvre, devienne non pas un autre, non pas du vivant qu'il était l'écrivain avec ses devoirs, ses satisfactions et ses intérêts, mais plutôt personne, le lieu vide et animé où retentit l'appel de l'œuvre" (Blanchot, Le Livre à Venir)

Cette citation est liée à la notion d' “ œuvre ” en tant qu'objet d'étude. Ceci permet à l'auteur de gloser sur le travail d'écriture et l'œuvre littéraire en tant que telle.

Quand il se réfère à Maurice Blanchot ou Albert Camus, le narrateur de Square Tolstoi les traite d'"oncle" ("tio"), ce qui dénote une proximité et une affection toutes particulières.

Fui outra vez direito ao busto, em passos pouco firmes. "Ouve isto", gritei eu depois de folhear o caderno. [...] "Pois aqui tens uma europeia mais recente, e do homem de esperanças que foi o tio Camus : 'Ce que l' intelligence et l' énergie de l' Europe ont fourni sans trêve à l' orgueil d' un temps misérable." Mais tarde - não está aqui, mas lembro-me - o Breton escreveu desta época que ela era un temps à ne pas mettre un chien dehors. Mas queres ouvir os bonzos do que o Bergamin chama a nova escolástica? Coisas assim : L'écriture ne fait plus signe de vérité. Pesquei isto no Tel Quel logo que cheguei a esta cidade. "(Bragança, 1981 : 211-212)

(fr.) Je suis retourné tout droit vers le buste, dans des pas mal assurés. "Écoute ceci", criais-je après avoir feuilleté mon cahier. [...] Eh bien en voici une européenne plus récente, et de l'homme d'espoir que fut l'oncle Camus: "Ce que l'intelligence et l'énergie de l'Europe ont fourni sans trêve à l'orgueil d'un temps misérable. "Plus tard - ce n'est pas ici, mais je m'en souviens - Breton a écrit de cette époque que c'était un temps à ne pas mettre un chien dehors. Mais tu veux entendre les bonzes que Bergamin nomme la nouvelle scolastique? Des choses du genre : L'écriture ne fait plus signe de vérité. J'ai péché ça dans le Tel Quel dès que je suis arrivé dans cette ville.

Cette joute verbale dénote également et surtout la proximité ressentie par le narrateur avec ces auteurs qu'il considère sans doute comme des maîtres à penser.

Finalement, Aníbal cite Denis de Rougemont, écrivain suisse connu notamment pour L'amour et l'Occident (1939), ouvrage qui porte sur la passion amoureuse : 
Levei para dentro da tina o Denis de Rougemont (L'amour et l'Occident). Abri ao calhas, e reli :

"Edgar Poe engendra Baudelaire qui engendra le symbolisme qui engendra des mandragores, des femmes sans corps, des jeunes Parques, des apparences à peine féminines de fuites - comme on dit que l'eau fuit d'un bassin : fissures dans le réel, fuites de rêves. "Fechei o livro e atirei-o ao lajedo sujo da casa de banho. "Tá bem, abelha, comentei. "Hoje não estou com físico para o teu paleio". (Bragança, 1981 : 191)

(fr.) J'ai emmené à l'intérieur de la baignoire Denis de Rougemont (L'amour et l'Occident). J'ai ouvert au hasard, et j'ai relu :

"Edgar Poe engendra Baudelaire qui engendra le symbolisme qui engendra des mandragores, des femmes sans corps, des jeunes Parques, des apparences à peine féminines de fuites - comme on dit que l'eau fuit d'un bassin : fissures dans le réel, fuites de rêves. " J'ai fermé le livre et je l'ai jeté sur le dallage sale de la salle de bains. "Très bien, espèce de fouine ", dis-je. "Aujourd'hui je ne suis pas d'humeur pour ton baratin".

Nous constatons que le narrateur dialogue littéralement avec cet auteur de façon familière.

À travers ces extraits nous détectons une grande proximité entre Aníbal, le narrateur de Square Tolstoi et ces références littéraires. De façon plus générale, nous pouvons affirmer que dans Square Tolstoi il existe un dialogue et une intertextualité inhérente à ce roman qui s'abreuve de références du monde littéraire français.

Les citations étrangères permettent une dimension réflexive du discours du narrateur et confèrent un cosmopolitisme et une universalité incontestables à ce roman. En ce qui concerne les citations et références artistiques, Aníbal cite deux répliques de deux acteurs de cinéma étrangers, Humphrey Bogart et Jean Seberg :

Nos intervalos duas figuras vinham-me fantasmear : Bogart, no final de $O$ Falcão de Malta, dizendo : "This is the kind of stuff dreams are made of." E a cara anja de Seberg, no final de À bout de Souffle, perguntando aos chuis: "Qu'est-ce que' degueulasse?' " [sic] (Bragança, 1981 : 182)

(fr.) Pendant les intervalles deux figures venaient me hanter : Bogart, à la fin du Faucon de Malte, en disant: "This is the kind of stuff dreams are made of." Et le visage d'ange de Seberg, à la fin de À bout de Souffle, demandant aux flics : "Qu'estce que "dégueulasse" " [sic] 
Ces répliques situées à la fin des deux films sont emblématiques pour le narrateur. Le narrateur de Square Tolstoi évoque également la chanson de Georges Moustaki "Il est trop tard" :

Sabe? A canção do Moustaki? 'Passe passe le temps, tu n'auras pas pour très longtemps.' E contudo ' il était encore temps.' Eu gostava - gosto ainda -tanto de você. (Bragança, 1981 : 183) (fr.) Vous connaissez? La chanson Moustaki? 'Passe passe le temps, tun' auras pas pour très longtemps.' Et pourtant ' il était encore temps.' Je vous aimais - je vous aime - encore tant.

Cette chanson est en liaison directe avec la thématique abordée par le narrateur concernant l'amour qu'il porte à sa compagne et la fin de leur relation.

Pour finir, la langue française est défendue et revendiquée par le narrateur. Comme le montre Aníbal qui prend position à l'OCDE à Paris à fin de prendre la parole en français,

Decidi falar em francês porque esta Organização tem sede em Paris, cidade que mais que uma vez proclamou desejos de uma República de Repúblicas. Ora já me aconteceu, no tal meio da noite parado em pleno túnel, entrever uma figura de mulher esplendorosa, e correr para ela convencido de estar frente ao corpo simbolizador de "Liberdade, Igualdade, Fraternidade". (Bragança, 1981 : 131)

(fr.) J'ai décidé de parler en français parce que cette Organisation siège à Paris, ville qui plus d'une fois a proclamé les désirs d'une République des Républiques. Or, il m'est déjà arrivé en pleine nuit d'entrevoir dans un tunnel une figure féminine splendide, je cours vers elle convaincu d'être en face du corps symbolisant la "Liberté, l'Égalité et la Fraternité ".

Le narrateur fait ainsi référence aux valeurs de la République Française car elles incarnent pour lui la liberté qu'il est venu chercher en France. Dans cette œuvre, nous sommes ainsi face à un plurilinguisme culturel qui contribue à un individualisme élitiste. Le plurilinguisme culturel du narrateur renforce le plurilinguisme littéraire et ses marques plurilingues. Nous remarquons que l'auteur, au niveau transtextuel, a généralement une préoccupation de contextualisation des extraits cités.

\section{Conclusions}

Le propos de cette étude a été de montrer que cette parole multiple, présente dans Square Tolstoi révèle une pratique d'écriture hétérogène et revêt un triple enjeu, linguistique, littéraire et culturel. En effet, les changements de langues contribuent à la conception globale 
de l'œuvre romanesque et l'influence. L'auteur est libre de choisir la langue de son œuvre, il peut créer ainsi sa langue romanesque. En changeant de langue - en tant que code linguistique - l'écrivain change aussi de public, car le rapport à la langue influence nécessairement la forme et le contenu de l'œuvre littéraire. Comme l'indique Lise Gauvin, écrire est un véritable "acte de langage":

Plus que de simples modes d'intégration de l'oralité dans l'écrit, ou que la représentation plus ou moins mimétique des langages sociaux, on dévoile ainsi le statut d'une littérature, son intégration/définition des codes et enfin toute une réflexion sur la nature et le fonctionnement du littéraire. (Gauvin, $2007: 113$ ).

Comme nous l'avons vu à travers notre analyse, la juxtaposition de citations et de termes en langue étrangère s'intercalent sans cesse dans la structure narrative du roman. Comme le remarque Dominique Combe, ceci participe de la polyphonie romanesque :

La polyphonie est le fait d'un plurilinguisme en acte; mais à la différence du bilinguisme, de traduction ou d'autotraduction, les langues s'y rencontrent non plus successivement, mais simultanément dans l'espace du livre (Combe, 1995 : 136-137).

Cette écriture plurilingue, avec des sources culturelles et linguistiques diverses, permet la construction d'un "métarécit identitaire " (cf. Bibeau, 1996) et attire l'attention sur la notion d'identité linguistique. Le plurilinguisme présent dans le roman représente une force centrifuge qui permet de décentrer et de rompre le discours monolingue et centralisé de l'État portugais. D' ailleurs, le titre du roman l'illustre à la perfection puisque Square Tolstoi fait à la fois référence au square du XVIo arrondissement de Paris et à l'auteur russe Léon Tolstoi.

En insérant la langue et la culture françaises dans son livre Nuno Bragança montre à quel point il est imprégné de la France. Son attachement francophile et francophone est, comme nous l'avons vu, très important et souligne sa prise de liberté par rapport au Portugal de l'époque. 


\section{Bibliographie}

BAKHTINE, Mikhaïl (1999, $1^{\text {ère }}$ éd. 1978). Esthétique et théorie du roman. Paris : Gallimard. BHABHA, Homi K (2007 $1^{\text {ère }}$ éd. en anglais 1994). Les lieux de la culture, une théorie postcoloniale. Paris : Payot.

BIBEAU, Gilles (1996). “Une identité en fragments. Une lecture ethnocritique du roman québécois ". In : Mikhaël Elbaz, Andrée Fortin, Guy Laforest (dirs.), Les frontières de l'identité. Sainte Foy : Presses de l'Université de Laval.

BISMUth, Hervé (2007). Le fou d'Elsa : métissages linguistiques et discursifs. Dijon :

Éditions Universitaires de Dijon.

BRAGanÇA, Nuno (1981). Square Tolstoï. Lisboa : Assírio e Alvim.

CALVET, Louis-Jean (1993). La sociolinguistique. Paris : P.U.F.

COMBE, Dominique (1995). Poétiques francophones. Paris : Hachette.

DERRIDA, Jacques (1996). Le monolinguisme de l'autre, ou, La prothèse d'origine. Paris : Galilée.

GAUVIN, Lise (2007). "Autor in fabula : pérégrinisme et paratexte ”. In : Danielle PerrotCorpet, Chrsitine Queffélec (éds.), Citer la langue de l'autre. Mots étrangers dans le roman, de Proust à W.G. Sebald. Lyon : Presses Universitaires de Lyon, pp.113-129.

KRISTEVA, Julia (1997). “L'autre langue ou traduire le sensible ”. In : Textuel, no 32, pp. 157170.

HALL, Stuart (2007). "La question multiculturelle ". In : Identités et cultures. Politique des "Cultural Studies", traduit de l'anglais par Christophe Jaquet et compilé par Maxime Cervulle. Paris : Éditions Amsterdam.

Huston, Nancy (1999). Nord Perdu suivi de Douze France. Paris : Actes Sud.

LEBLANC, Julie (2006) (dir.) “L'Autobiographique ”. In : Texte, Revue de critique et de théorie littéraire, $\mathrm{n}^{\circ}$ 39/40.

LeVI-Strauss, Claude (1983). Le regard éloigné. Paris : Plon.

MARQUES, Isabelle Simões (2009). Le plurilinguisme dans le roman portugais contemporain (1963-1983) : caractéristiques, configurations linguistiques et énonciatives. Thèse de Doctorat : Université Paris 8- Université Nouvelle de Lisbonne.

RIC@EUR, Paul (2004). Sur la traduction. Paris : Bayard.

ROANI, Gerson Luiz (2004). «Sob o vermelho dos cravos de Abril - literatura e revolução no Portugal contemporâneo “. In : Revista Letras, Curitiba, no 64, pp. 15-32.

SEIXO, Maria Alzira (1983). «Resenha sobre Square Tolstoï de Nuno Bragança”. In :

Colóquio- Letras, nำ71, pp. 96-98.

STEINER, Georges (2003). Extratérritorialité, Essai sur la littérature et la révolution du langage. Paris : Hachette Littératures. 
